\title{
Science cases for the OWL Earth-like planet imager and spectrograph (EPICS)
}

\section{J.-L. Beuzit ${ }^{1}$, R. Gratton ${ }^{2}$, M. Kasper ${ }^{3}$, S. Desidera ${ }^{2}$, F. Kerber ${ }^{3}$,} F. Rahoui ${ }^{3}$, D. Mouillet ${ }^{4}$, D. Rouan $^{5}$, M. Turatto ${ }^{2}$, M. Feldt $^{6}$,

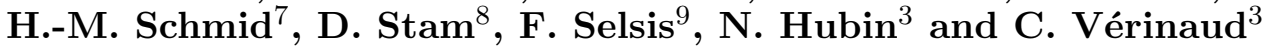

${ }^{1}$ Laboratoire d'Astrophysique de Grenoble, BP 53, F-38041 Grenoble Cedex 9, France email: Jean-Luc.Beuzit@obs.ujf-grenoble.fr

\footnotetext{
${ }^{2}$ Osservatorio Astronomico di Padova, Vicolo dell'Osservatorio 5, I-35122 Padova, Italy

${ }^{3}$ European Southern Observatory, Karl-Schwarzschild-Strasse 2, D-85748 Garching, Germany

${ }^{4}$ Laboratoire d'Astrophysique de Toulouse et Tarbes, BP 826, F-65008 Tarbes Cedex, France

${ }^{5}$ Laboratoire d'Etudes Spatiales et d'Instrumentation Astrophysique, F-92190 Meudon, France

${ }^{6}$ Max-Planck-Institut für Astronomie, Königstuhl 17, D-69117 Heidelberg, Germany

${ }^{7}$ Institut für Astronomie, ETH Zentrum, CH-8092 Zurich, Switzerland

${ }^{8}$ Universiteit van Amsterdam, Kruislaan 403, NL-1098 Amsterdam, The Netherlands

${ }^{9}$ Ecole Normale Supérieure de Lyon, F-69364 Lyon Cedex 07, France
}

\begin{abstract}
The extreme contrast in mass and luminosity between the extra-solar planets and their host stars make detailed studies of these planets very challenging. In particular, direct observations of extra-solar planets is still beyond the capabilities of the currently available instrumentation, save for perhaps a few extreme cases of very young and massive planets at large distances from the central star. While progress in instrumentation might allow significant progress in detection capabilities either with the 8 and 10-m ground-based telescopes (Planet Finder instruments on the VLT and Gemini) or with the next generation space telescope (JWST), imaging of extra-solar planets over a wide range of parameters, and possibly down to terrestrial planets, will require extremely large ground-based telescopes like OWL or dedicated space instrumentation (TPF or Darwin for instance). We outline here the scientific objectives of EPICS, the OWL Earth-like Planet Imager and Spectrograph, summarize the corresponding high level requirements, present the foreseen observing modes and give a first estimate of its performance.
\end{abstract}

Keywords. instrumentation: adaptive optics — techniques: high angular resolution — planetary systems - planetary systems: formation

\section{Introduction}

One of the most ambitious science objectives of the European Southern Observatory (ESO) OverWhelmingly Large (OWL) telescope project will be the detection and characterization of extra-solar systems in an advanced evolutionary stage, for a statistically meaningful sample of stars. In particular, rocky planets with possibly Earth-like features will be the ultimate and most challenging goal of such a programme.

ESO has carried out, with the collaboration of scientists and engineers from several European institutes already involved in the VLT Planet Finder project for the VLT (Beuzit et al. 2006), a preliminary 6-month study of a possible Earth-like Planet Imager and Spectrograph (EPICS) instrument for OWL. The EPICS concept has been naturally biased but also inspired by the VLT Planet Finder feasibility studies. These studies have demonstrated that it is necessary to combine an "eXtreme" Adaptive Optics system 
(XAO) with other methods (coronagraphy and differential detection techniques) to reach the contrast required for extra-solar planets detection.

\section{Science objectives}

Direct imaging of extra-solar planets is of extreme importance for a number of reasons: (i) acquisition of their images would provide immediate confirmation of their existence; (ii) analysis of their spectra would provide direct information about the presence and composition of their atmospheres, and even ultimately about the presence of life on their surface; (iii) analysis of their polarization characteristics can provide clues on the structure of their atmospheres (presence of dust); (iv) determination of their positions at different epochs would provide their orbit; (v) perturbations around an elliptical orbit might disclose the presence of other unseen bodies in the system (like for the discovery of Neptune in our own Solar System); (vi) analysis of the light curve might provide the period of rotation of the planet around its own axis, information about the presence of satellites and rings, and finally data about weather or even about the presence of continents covered by vegetation. It is therefore clear that our knowledge of extra-solar planets can only really progress into a mature stage of detailed characterization through direct imaging techniques.

Direct detection of extra-solar planets is however made very difficult by the very high relative flux ratio between the host star and the planets orbiting it and the small angular separation between them. Ultimately the primary science goal of EPICS, the detection of rocky planets with Earth-like features, will require the detection of faint point sources in proximity of a bright star with an object-star contrast down to about $2 \times 10^{-10}$ at $0.05^{\prime \prime}$ from the host star. Moreover, to observe a planet and to characterize its atmosphere, EPICS will have to be sensitive at the wavelengths of $\mathrm{H} 2 \mathrm{O}, \mathrm{CO}_{2}, \mathrm{CH}_{4}$ and $\mathrm{O}_{2}$ molecular absorption lines. EPICS will also permit a significant breakthrough in the detection and characterization of cold gas giant planets. Its improved capabilities in terms of contrast and resolution (allowing a better separation) when compared to existing or foreseen instruments for $8 \mathrm{~m}$ class telescopes will permit an easier detection of these giant planets, and will also open the door to high resolution spectroscopy. In particular, radial velocity measurements and the analysis of atmospheric composition and dynamics of close-in giant planets will be possible.

\section{High level requirements}

The key scientific requirements derived from the science analysis and driving the design of the instrument are summarized below:

- EPICS shall be sensitive to wavelengths from 0.6 to $1.75 \mu \mathrm{m}$. For the detection of terrestrial planets, the wavelength range 700 to $900 \mathrm{~nm}$ will be the most interesting, especially with its $\mathrm{O}_{2}$ band. Spectra of gas giant planets are dominated by the methane features in $J$ - and $H$-bands. Wavelengths shorter than $700 \mathrm{~nm}$ might be interesting because of a higher degree of polarization.

- The total field-of-view of EPICS in all observing modes shall be at least $2^{\prime \prime}$ in diameter at visible wavelengths, corresponding to $1 \mathrm{AU}$ at $1 \mathrm{pc}$, i.e. large enough to cover terrestrial planets at all distances, and $4^{\prime \prime}$ in diameter in the near-infrared, accounting for the larger separation at which giant planets are searched for (the $4^{\prime \prime}$ field allows to cover the Solar System, apart from Neptune and Pluto, at distances larger than 10pc). 


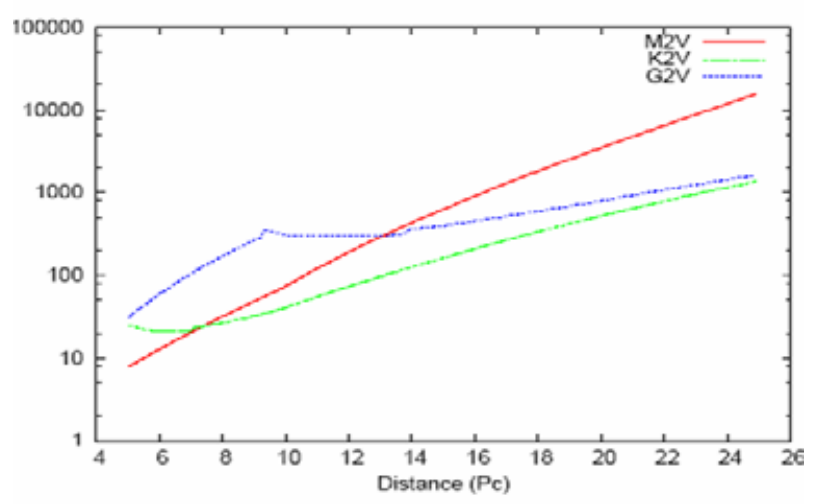

Figure 1. Exposure time (in hours) needed to detect $\mathrm{O}_{2}$ (at $760 \mathrm{~nm}$ ) at $5 \sigma$ on an Earth-like planet orbiting a Main Sequence star, as a function of the distance to the host star. The three curves correspond to different spectral types $(\mathrm{t} 0=4 \mathrm{~ms}, \mathrm{r} 0=20 \mathrm{~cm}, \mathrm{~T}=16 \%, \mathrm{R}=150)$.

- The inner working angle for all observing modes operating at visible wavelengths shall be smaller than 30 mas, corresponding to $0.3 \mathrm{AU}$ at $10 \mathrm{pc}$, i.e. small enough to cover the Solar System at $10 \mathrm{pc}$ or to resolve the Earth out to $25 \mathrm{pc}$.

- The spatial sampling shall fulfil at least the Nyquist criterion at all wavelengths.

- The contrast versus separation requirements (to search for terrestrial planets in habitable zone) shall be respectively: $2 \times 10^{-10}$ at 40 mas ( $\mathrm{G}$ star at $25 \mathrm{pc}$ ), $8 \times 10^{-10}$ at $25 \mathrm{mas}$ (K star at $20 \mathrm{pc}$ ) and $8 \times 10^{-9}$ at $15 \mathrm{mas}$ (M star at $15 \mathrm{pc}$ ).

- The relative astrometric precision for all main observing modes shall be better than $100 \mu$ arcsec with a goal at $10 \mu$ arcsec, to allow for the determination of the main orbital parameters of the planets and possibly the detection of unseen satellites by photocenter wobble in very favorable cases, for almost equal mass planets and satellites.

- The absolute/relative photometric precision for all main observing modes shall be better than $1 \%$, to allow for observations of planetary rotation, weather and phase variations, satellites (transit and eclipses), etc.

- The adaptive optics control radius shall be larger than $0.4^{\prime \prime}$ at $800 \mathrm{~nm}$, corresponding to about $1 \mathrm{AU}$ at $2.5 \mathrm{pc}$, and therefore ensuring that the prime targets are inside the control radius. This would then correspond to an actuator pitch of $\sim 0.2 \mathrm{~m}$.

- The adaptive optics wavefront sensor limiting magnitude shall allow to observe at least 100 stars of G, K and M spectral types. This typically corresponds to $\mathrm{m}_{I} \sim 9$.

- Simultaneous search for terrestrial and gas giant planets shall be possible.

\section{Observing modes and performance}

A detailed description of the EPICS concept is outside the scope of this paper (see Monnet 2006, Fusco, et al. 2006, these proceedings). We list hereafter the main observing modes with their specific interest. Each of these scientific channels will be equipped with its own coronagraph. The three modes can be operated simultaneously for maximizing the scientific return.

- The $R$-band will be dedicated to the Polarimetric Differential Imager for detection of rocky planets and to the follow-up observations for the detection of $\mathrm{O}_{2}$. 


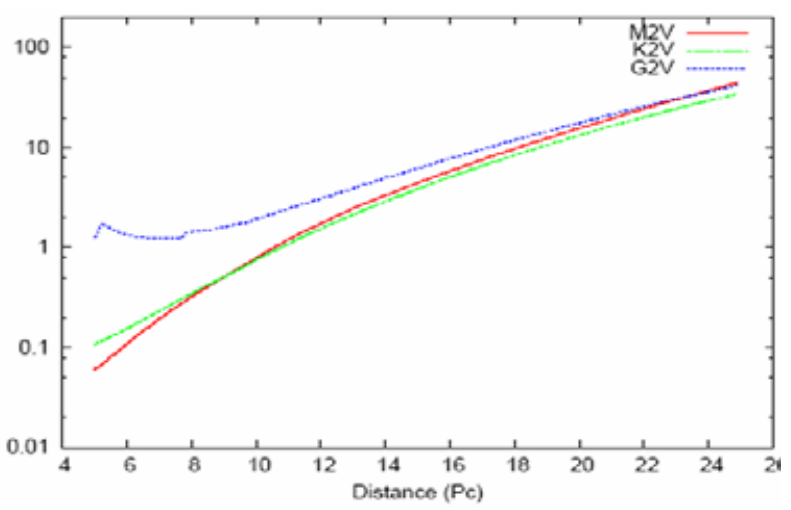

Figure 2. Exposure time (in hours) needed to detect $\mathrm{H}_{2} \mathrm{O}(1.25 \mu \mathrm{m})$ at $50 \sigma$ on a Jupiter-like planet orbiting a Main Sequence star, as a function of the distance to the host star. The three curves correspond to different spectral types $(\mathrm{t} 0=4 \mathrm{~ms}, \mathrm{r} 0=20 \mathrm{~cm}, \mathrm{~T}=16 \%, \mathrm{R}=15)$.

\begin{tabular}{|c|c|c|}
\hline & Integration & (Hours) \\
\hline $\begin{array}{c}\text { Earth-like planet } \\
\mathbf{H}_{2} \mathbf{O} \\
\text { (J band, } \mathrm{R}-15)\end{array}$ & $\begin{array}{l}\mathrm{G} 2 \text { at } 25 \mathrm{po} \\
\mathrm{K} 2 \text { at } 20 \mathrm{pc} \\
\mathrm{M} 2 \text { at } 15 \mathrm{pe}\end{array}$ & $\begin{array}{l}30 \\
15 \\
4\end{array}$ \\
\hline $\begin{array}{l}\text { Earth-sized planet } \\
\mathrm{CO}_{2} \text { rich }(10 \%) \\
\text { ( } \mathrm{H} \text { band } \mathrm{R}=15)\end{array}$ & $\begin{array}{l}\mathrm{G} 2 \text { at } 25 \mathrm{pc} \\
\mathrm{K} 2 \text { at } 20 \mathrm{pc} \\
\mathrm{M} 2 \text { at } 15 \mathrm{pe}\end{array}$ & $\begin{array}{c}70 \\
30 \\
5\end{array}$ \\
\hline $\begin{array}{l}\text { Earth-like planet } \\
\mathbf{O}_{2} \\
\text { (R band. } \mathrm{R}-150 \text { ) }\end{array}$ & $\begin{array}{l}\mathrm{G} 2 \text { at } 25 \mathrm{po} \\
\mathrm{K} 2 \text { at } 20 \mathrm{po} \\
\mathrm{M} 2 \text { at } 15 \mathrm{pc}\end{array}$ & $\begin{array}{c}15000 \\
500 \\
800\end{array}$ \\
\hline $\begin{array}{l}\text { Earth-like planet } \\
\text { Pola. 15\% } \\
(600-800 \mathrm{~nm})\end{array}$ & $\begin{array}{l}\mathrm{G} 2 \text { at } 25 \mathrm{po} \\
\mathrm{K} 2 \text { at } 20 \mathrm{po} \\
\mathrm{M} 2 \text { at } 15 \mathrm{pe}\end{array}$ & $\begin{array}{c}2000 \\
60 \\
150\end{array}$ \\
\hline $\begin{array}{l}\text { Jupiter-like planet } \\
\text { CH, } \\
\text { (H band, R }=15 \text { ), SNR }=50\end{array}$ & $\begin{array}{l}\mathrm{G} 2 \text { at } 25 \mathrm{po} \\
\mathrm{K} 2 \text { at } 20 \mathrm{pe} \\
\mathrm{M} 2 \text { at } 15 \mathrm{pe}\end{array}$ & $\begin{array}{c}40 \\
2 \\
1.5 \\
\end{array}$ \\
\hline
\end{tabular}

Figure 3. Exposure times needed to detect various classes of extra-solar planets with EPICS. SNR is 5 for Earth-like planets and 50 for giant planets.

- The $J$-band will be equipped with a differential imager using pairs of filters that will be sensitive to $\mathrm{CH}_{4}$ and $\mathrm{H}_{2} \mathrm{O}$ absorption bands.

- The $H$-band will be equipped with an Integral Field Spectrograph, mainly targeted to the detection of $\mathrm{CH}_{4}$ and $\mathrm{CO}_{2}$.

The $I$-band has been reserved for wave-front sensing because of its lower scientific interest for planet detection. Its spectral location, between the visible and near-infrared instruments, is optimal with respect to the important atmospheric chromatic limitations for extreme adaptive optics systems on ELTs.

Figures 1 and 2 illustrate the EPICS expected performances for the detection of terrestrial and gas giant planets. The exposure time needed to detect respectively an Earth-like planet and a Jupiter-like planet around Main Sequence stars of various spectral types is given as a function of the distance of the host star. Figure 3 presents a summary of the exposure times needed for different cases of planets, observing modes and distances.

\section{References}

Beuzit, J.-L., Feldt, M., Mouillet, D., Moutou, C., Dohlen, K., Puget, P. et al. 2006, in: C. Aime, F. Vakili (eds.), Direct Imaging of Exoplanets: Science \& Techniques, IAU Colloquium 200, in press 
Fusco, T., Vérinaud, C., Rousset, G., Beuzit, J.-L., Kasper, M., Mouillet, D. \& Hubin, N. 2006, these proceedings

Monnet, G. 2006, these proceedings

\section{Discussion}

ZINNECKER: Are your calculations specific to OWL only or are they also relevant for say a $50 \mathrm{~m}$ telescope?

BEUZIT: Our calculations are not specific to OWL and they can very easily be adapted to different telescope sizes. To first order, the scaling can be obtained by applying the $T_{\text {out }} \propto D^{-4}$ relation. 